hypertension among 1424 men, on CRP among 1422 men and 2466 women and on metabolic syndrome among 1710 men and 2896 women, and a prospective study on type 2 diabetes among 1603 men and 2795 women.

Results Compared with no NIH, the multivariable OR of hypertension was 1.63 (95\% CI 1.1 to 2.5) for moderate-to-severe NIH. The prevalence of a high CRP ( $\geq 1.0 \mathrm{mg} / \mathrm{l}$ ) was 1.4 to 1.7 -fold higher for mild to severe NIH in both sexes. The multivariable ORs of metabolic syndrome was 3.2 (2.2 to 4.7) for moderate-to-severe NIH among men and 5.8 (3.4 to 9.8) among women, respectively. The multivariable-adjusted HRs of developing type 2 diabetes was 1.3 (0.9 to 1.8) among those with mild NIH and 1.7 (1.0 to 2.8) among those with moderate-to-severe NIH.

Conclusion NIH was associated with hypertension, elevated serum CRP levels, the accumulation of metabolic risk factors, and increased risk of developing type 2 diabetes among middle-aged Japanese.

\section{P2-303 DEVELOPMENT OF PREDICTIVE EQUATIONS FOR DXA MEASURES OF ADIPOSITY IN AN INDIAN POPULATION}

doi:10.1136/jech.2011.142976k.36

${ }^{1} \mathrm{~A}$ Taylor, ${ }^{2} \mathrm{H}$ Kuper, ${ }^{3} \mathrm{~J}$ Wells, ${ }^{4} \mathrm{~K}$ V Radhakrishna, ${ }^{2} \mathrm{~S}$ Kinra, ${ }^{1} \mathrm{~N}$ Timpson, ${ }^{4} \mathrm{~B}$ Kulkarni, ${ }^{1} \mathrm{G}$ Davey-Smith, ${ }^{2} \mathrm{~S}$ Ebrahim, ${ }^{1} \mathrm{Y}$ Ben-Shlomo. ${ }^{1}$ University of Bristol, Bristol, UK; ${ }^{2}$ London School of Hygiene and Tropical Medicine, London, UK; ${ }^{3}$ Institute of Child Health, London, UK; ${ }^{4}$ National Institute of Nutrition, Hyderabad, India

Introduction Predictive equations for DXA measures of total and abdominal adiposity from simple anthropometry for an Indian population will enable more accurate estimates of adiposity to be derived in other studies.

Methods Using 1230 men and 636 women from Hyderabad, India, multiple linear regression was performed to generate predictive equations for DXA measures of total body fat (grams) and total abdominal fat in the L1-L4 intervertebral region (grams). Equations were developed separately for each sex on a training set $(60 \%$ of sample) and tested on the validation set ( $40 \%$ of sample).

Results For total body fat in males, a simple equation based on two variables (height and weight) gave an $\mathrm{R}^{2}$ of 0.83 and SEE (SE of the estimate) (square root of the sum of observed- predicted values/ number of observations) of $2200 \mathrm{~g}$ whereas a more complex equation (additionally including triceps skinfold, waist circumference and calf skinfold) gave an $\mathrm{R}^{2}$ of 0.94 ( $\mathrm{SEE}=1600 \mathrm{~g}$ ). In females, hip circumference and calf skinfold alone explained $92 \%$ of the variance in total body fat (SEE $2300 \mathrm{~g}$ ) increasing to $95 \%$ with waist circumference, subscapular skinfold, weight and calf circumference included (SEE $1800 \mathrm{~g}$ ). Waist circumference was the best predictor of fat in the $\mathrm{L} 1-\mathrm{L} 4$ region for both sexes $\left(\mathrm{R}^{2}=0.88\right.$ and 0.89 respectively); more complex equations achieved an $\mathrm{R}^{2}$ of $93 \%$. Predictive equations for both traits produced an $\mathrm{SEE}<0.5 \mathrm{SDs}$, indicating good accuracy.

Conclusion DXA measures of adiposity can be derived with a high degree of precision from simple anthropometric measures in an Indian population.

\section{P2-304 CHILDHOOD INFECTIOUS DISEASES AND PREMATURE ADULT MORTALITY: RESULTS FROM THE NEWCASTLE THOUSAND FAMILIES STUDY}

doi:10.1136/jech.2011.142976k.37

${ }^{1} \mathrm{P}$ Tennant, ${ }^{2} \mathrm{~L}$ Parker, ${ }^{1} \mathrm{M}$ Pearce. ${ }^{1}$ Institute of Health and Society, Newcastle University, Newcastle upon Tyne, UK; ${ }^{2}$ Department of Medicine, Pediatrics, Dalhousie University, Nova Scotia, Canada

Introduction Early life infections may negatively influence health in later life, but there have been few studies of the association, due to the difficulty of obtaining adequate information from throughout life. This study utilised longitudinal data from the Newcastle Thousand Families Study, a prospective cohort of 1147 individuals born in Newcastle-upon-Tyne (UK) in 1947, to assess the impact of various childhood infectious diseases on mortality between ages 18 and 60 years.

Methods Detailed information was collected prospectively at birth and during childhood on a number of early life factors. Study members were "flagged" by the UK National Health Service Central Register when they died or emigrated. Death between ages 18-60 years was analysed in relation to childhood infections, adjusting for potential confounders, using Cox regression.

Results History of infection with either tuberculosis or whooping cough was independently predictive of mortality between ages 18 and 60 years [Adjusted HR, aHR=2.00 (95\% CI 1.17 to 3.41) and $1.95(95 \%$ CI 1.21 to 3.14$)$ respectively]. Of the other variables examined, adult mortality was more common among men $[\mathrm{aHR}=0.62(95 \% \mathrm{CI} 0.39$ to 0.97$)]$ and among first-borns $[\mathrm{aHR}=2.95$ (95\% CI 1.52 to 5.73$)$ ]. The effect of whooping cough on mortality was largely attributable to a higher risk of death from cancer, particularly non-smoking related cancers.

Conclusion In a pre-vaccination cohort from Northern England (UK), childhood infection with tuberculosis or whooping cough was associated with an increased risk of premature adult mortality independent of other childhood circumstances. Further studies are required to investigate this association in different populations.

\section{P2-305 FIVE-YEAR CHANGES IN DIETARY INDEXES ARE ASSOCIATED WITH CHANGES IN CARDIOVASCULAR RISK FACTORS}

doi:10.1136/jech.2011.142976k.38

U Toft, ${ }^{*}$ T Jørgensen. Research Centre for Prevention and Health, Glostrup, Denmark

Rationale and Objectives The sensitivity of dietary indexes to measure changes in dietary habits is unclear. The objective of the study was to investigate whether 5 -year changes in the Dietary Quality Score (DOS), Healthy Eating Index, Dietary Quality Index (DOI) and the Mediterranean diet score were associated with changes in dietary related cardiovascular (CVD) risk factors.

Materials and Methods A random sample of 13016 men and women aged 30-60 years were invited for a 5-year lifestyle intervention programme. A total of 4562 were included in the statistical analysis. The association between changes in dietary indexes and changes in CVD risk factors was investigated in multiple linear regression models.

Results and Findings Improved dietary habits according to the DOS at 5-year follow-up was significant negatively associated with changes in weight $(p=0.01)$, waist circumference $(p=0.009)$, total cholesterol $(p=0.02)$, LDL cholesterol $(p=0.006)$. The Mediterranean diet score was significantly associated with weight $(p=0.001)$ and diastolic blood pressure $(p=0.02)$, the DOI was borderline significant associated with weight and waist circumference, whereas no significant association was found for the Healthy Eating Index.

Conclusion Changes in the simple DOS were stronger associated with changes in CVD risk factors than the more complex dietary indexes. 